\title{
Treatment of rheumatoid arthritis with interleukin 1 receptor antagonist
}

\author{
Barry Bresnihan
}

Rheumatoid arthritis (RA) is characterised by chronic synovial inflammation of multiple large and small joints in the upper and lower limbs. ${ }^{1}$ The pathogenesis of RA is complex and involves the activation of several synovial cell populations and the production of many proinflammatory and destructive mediators including cytokines, prostaglandins and proteases. Many investigators have suggested that tumour necrosis factor (TNF) and interleukin 1 (IL1) play a critical part in the pathogenesis of RA. ${ }^{2}$ The IL1 family includes IL $1 \alpha$, IL $1 \beta$ and IL1 receptor antagonist (IL1Ra). ${ }^{3}$ IL $1 \alpha$ and IL1 $\beta$ are both agonist molecules that can influence the functions of most cell types by binding to the IL1 receptor (IL1R). In experimental models of arthritis IL1 $\beta$ causes exacerbation of disease, stimulates cartilage and bone resorption and inhibits the synthesis of articular collagen and proteoglycan. ${ }^{4}$ IL $1 \mathrm{Ra}$ is responsible for the regulation of IL $1 \alpha$ and IL $1 \beta$ by competitively binding with high avidity to IL1R. Thus, the level of target cell activation may depend on the balance between the agonistic effect of IL $1 \alpha$ and IL $1 \beta$ and the inhibitory effect of IL1Ra, each competing for the same cell surface receptor. ${ }^{23}$

IL1 Ra has been given therapeutically in several experimental models of arthritis with dramatic effects. ${ }^{4}$ For example, IL1Ra was administered intraperitoneally by continuous infusion to male $\mathrm{DBA} / 1$ mice with collagen induced arthritis (CIA). ${ }^{5}$ This resulted in clear suppression of established arthritis. Histological analysis demonstrated markedly reduced cartilage destruction. Moreover, the synthetic function of articular cartilage chondocytes fully recovered. Further studies of IL1 inhibition in experimental arthritis have confirmed the dominant role of IL 1 in cartilage destruction and have established the hypothesis that optimal IL1 blockade will result in reduced joint inflammation and the prevention of progressive joint damage. ${ }^{4}$

Further insights into the potential merits of IL1 Ra treatment have been provided by studies of gene therapy. Thus, using the murine CIA model, fibroblasts transfected with the IL1Ra gene were transplanted into the knee joint cavity before the onset of arthritis. ${ }^{6}$ Arthritis was almost completely prevented in the joints containing IL1Ra producing cells. In contrast, joints containing normal cells demonstrated severe inflammation and cartilage destruction. Moreover, the onset of arthritis in the ipsilateral paws of the IL1Ra gene bearing knee joint was also prevented.

\section{Interleukin 1 blockade in rheumatoid arthritis}

SOLUBLE IL1 RECEPTOR

Both IL $1 \alpha$ and IL $1 \beta$ mediate their biological activity through two specific membrane receptors, designated type I and type II. ${ }^{3}$ Soluble forms of both receptors have been detected in human body fluids. A recombinant soluble human form of type I IL1R ( $r H u I L 1 R I)$ was produced. $^{7}$ Quantitative binding studies demonstrated that the affinity of rHuIL1RI was equivalent to the full length membrane bound IL1RI.

Twenty three patients were enrolled into a randomised dose ranging, double blind, placebo controlled study of rHuIL1RI, administered subcutaneously for 28 consecutive days. The doses were $125,250,500$ or $1000 \mathrm{mg} / \mathrm{m}^{2} /$ day. Treatment with rHuIL1RI resulted in a reduction of monocyte cell surface IL $1 \alpha$, which indicated that the doses used were functional. However, only one patient, who received the highest concentration of rHuIL1RI, demonstrated clinically relevant improvement, and dose limiting toxicity was observed in two. Further studies of treatment with soluble IL1R have not been reported.

IL1RA

Dose range study

One hundred and seventy five patients with active RA were enrolled in a randomised, double blinded trial of recombinant human IL1Ra administered by subcutaneous injection. ${ }^{8}$ The study was divided into two phases. During the initial three week treatment phase, patients received IL1Ra 20, 70 or $200 \mathrm{mg}$ once, three times or on several days per week. A second four week maintenance phase followed immediately when patients received their treatment phase dose once weekly. Treatment was generally well tolerated. Injection site reactions were the most frequently reported adverse event observed in $62 \%$ of patients. Only 5\% withdrew prematurely because of an injection site reaction. Six per cent withdrew because of unsatisfactory therapeutic response. Dropout rates were similar across dose levels and dosing frequencies. Infections thought to be of bacterial origin were observed in $8 \%$ of patients who received IL1Ra $20 \mathrm{mg} /$ day, 11\% receiving 70 $\mathrm{mg} /$ day and $13 \%$ receiving $200 \mathrm{mg} /$ day.

Because of the lack of a placebo arm and the multiple small treatment groups, a definitive statement regarding the efficacy of IL1Ra was not possible. However, at the end of the three week treatment phase daily dosing appeared more effective than weekly dosing when assessed by the number of swollen joints $(\mathrm{p}=0.0001$, investigator $(\mathrm{p}=0.0002)$ and pa-
Correspondence to: Professor B Bresnihan.
Department of Vincents University Hospital, Dublin 4, Ireland 
tient assessment of disease activity $(\mathrm{p}=0.001)$, pain score $(p=0.0007)$ and $C$ reactive protein $(\mathrm{CRP})$ concentrations $(\mathrm{p}=0.0005)$. It was concluded that IL1 Ra could be safely administered by subcutaneous injection to patients with RA. The frequency of dosing seemed to be important in determining the clinical response, with daily administration providing the most benefit.

\section{Randomised clinical trial}

After the observations of the dose range study, a total of 472 patients were recruited into a randomised, 24 week, double blind, placebo controlled multicentre study. ${ }^{9}$ Patients were randomised into one of the four treatment groups: placebo or a single self administered subcutaneous injection of IL1 Ra $30 \mathrm{mg}, 75 \mathrm{mg}$ or $150 \mathrm{mg} /$ day. Patients ranged in age from 18 to 75 years and all met the American College of Rheumatology (ACR) criteria for the diagnosis of RA, had symptoms for $>6$ months and $<8$ years. The clinical parameters of disease activity were similar in the treatment groups and were consistent with active and severe RA: mean number of swollen joints 25.6 to 26.6, number of tender joints 32.8 to 35.7 , erythrocyte sedimentation rate (ESR) 46.8 to 53.2 $\mathrm{mm} 1 \mathrm{st} \mathrm{h}$, CRP 4.0 to $4.2 \mathrm{mg} / \mathrm{dl}$. In each treatment group, between 69.0 and $76.5 \%$ of patients had radiographic erosions.

The primary outcome measure was the ACR $20 \%$ response. $^{10}$ The response in the placebo group was relatively high at $27 \%$. However, the response in the IL1Ra $150 \mathrm{mg} /$ day group was significantly higher at $43 \%(\mathrm{p}=0.014)$. Forty four per cent of the IL1 Ra $150 \mathrm{mg}$ /day group satisfied the Paulus criteria ${ }^{11}$ for a $20 \%$ improvement $(p=0.0003)$. Overall, those who received IL1Ra $150 \mathrm{mg} /$ day demonstrated the maximal responses compared with placebo at 24 weeks: number of swollen $(p=0.009)$ and tender joints $(p=0.0009)$, investigator $(p=0.0006)$ and patient assessment of disease activity $(\mathrm{p}=0.012)$, pain VAS $(\mathrm{p}=0.001)$, HAQ score $(p=0.0007)$, ESR $(p<0.0001)$ and CRP $(p=0.0017)$. The clinical effect became apparent at approximately four weeks after starting treatment whereas the laboratory effects were evident at one week.

IL1Ra was generally well tolerated during the 24 weeks. As in the dose range study, the most frequent adverse event was an injection site reaction, experienced by $25 \%$ of the placebo group and by $50 \%, 73 \%$ and $81 \%$ of patients in the IL1Ra 30, 75 and $150 \mathrm{mg} /$ day groups, respectively. The reactions were usually mild and transient. Only $2 \%$ of the placebo group withdrew from the study because of injection site reactions, compared with $<1 \%$, $3 \%$ and $5 \%$ of patients in the IL1Ra, 30, 75 and $150 \mathrm{mg} /$ day groups, respectively. Other adverse events were encountered infrequently and affected the placebo group as frequently as the IL1Ra groups. Thus, both the efficacy and the safety of IL1Ra was confirmed in a large cohort of patients with active and severe RA.
Extension study

The patients who completed the 24 week randomised clinical trial continued treatment for an additional 24 weeks in a double blind, nonplacebo controlled extension study. ${ }^{12}$ Patients who had received placebo were randomised into one of the three different treatment arms and demonstrated clinical responses similar to those observed during the first 24 weeks of treatment. Those who had received IL1Ra during the first 24 weeks continued their treatment dose and maintained their clinical improvement. Long term treatment with IL1Ra was well tolerated.

\section{Effects on joint damage}

Serial hand radiographs obtained immediately before and after 24 weeks of treatment were available from 347 of the 472 patients $(74 \%)$ entering the randomised clinical trial. ${ }^{9}$ Radiographs were analysed according to the Larsen method. ${ }^{13}$ The mean Larsen score in the placebo group increased by 6.4 , from 15.4 at baseline to 21.8 at 24 weeks. In contrast, the mean Larsen score in the patients receiving IL1 Ra increased by only 3.8. This represents a $41 \%$ reduction in the rate of radiographic joint damage over 24 weeks ( $p=0.03$ ). The effects on radiographic joint damage was similar in the three treatment groups: $44 \%, 39 \%$ and $37 \%$ reduction in the rate of radiographic joint damage compared with placebo in the IL1Ra 30,75 and $150 \mathrm{mg} /$ day groups, respectively. When radiographic joint damage was measured according to the number of joints with erosions, an increase of 2.6 from 5.0 to 7.6 was observed in the placebo group. In contrast, the mean number of joints with erosions in the IL1Ra treated groups increased by only 1.4 $(p=0.004$ compared with placebo), representing a reduction of $46 \%$.

A further analysis of radiographic change was performed using the Genant method, ${ }^{14}$ which distinguishes two aspects of joint damage: articular erosion, representing direct invasion of cartilage and bone by proliferating synovial pannus, and joint space narrowing, which represents diffuse enzymatic destruction of the cartilage surfaces. Radiographs obtained before and after the randomised clinical trial and after the 24 week extension study were analysed. There were strong correlations between the Genant scores and the Larsen scores at each time point. The analyses produced a number of additional interesting observations. Firstly, patients who received IL1 Ra during the 0-24 week period demonstrated a $58 \%$ reduction in the rate of radiographic joint space narrowing compared with placebo $(p=0.003)$ but only a $38 \%$ reduction in the rate of radiographic erosion $(p=0.01)$, suggesting that the predominant early manifestation of blocking IL1 mediated joint damage is a reduction in the rate of cartilage surface loss. Secondly, when patients who received IL1Ra both during the 0-24 week period and the 24-48 week period were analysed it was observed that the mean changes in the measures of joint space narrowing according to the Genant scoring method were identical $(0.61)$ in the two treatment peri- 
ods. In contrast, the mean changes in the measures of joint erosion were 1.22 during the 0-24 week period but only 0.58 in the $24-48$ week period $(p=0.0001)$. This suggests that blocking IL1 mediated cartilage and bone invasion in vivo is achieved more slowly. To augment the effect of IL1 Ra treatment on joint damage it will be necessary to optimise delivery systems. ${ }^{15}$ In experimental arthritis the maximal effects were observed when IL1Ra was administered by continuous infusion. ${ }^{4}$

Effects on synovial tissue

Twelve paired synovial biopsy samples were obtained before and after entering the randomised clinical trial of IL1Ra. ${ }^{16}$ There was a notable reduction in intimal layer macrophage accumulation and subintimal macrophage and lymphocyte infiltration after IL1Ra $150 \mathrm{mg} /$ day compared with placebo and IL1Ra 30 $\mathrm{mg}$ /day. Down regulation of $\mathrm{E}$ selectin, vascular cell adhesion molecule 1 and intercellular adhesion molecule 1 was also associated with IL1 Ra $150 \mathrm{mg} /$ day. Apparent total arrest of joint damage was observed in four patients and all had received IL1Ra. The arrest of joint damage was associated with the cessation or reversal of intimal layer macrophage accumulation. These preliminary observations may represent inhibition of several biologically relevant IL1 mediated pathogenic effects.

\section{Conclusion}

Experimental studies have demonstrated that IL1 blockade with either anti-IL1 antibodies or IL1Ra produced significant, but often modest, anti-inflammatory effects and potent inhibition of cartilage and bone damage. Maximal effectiveness of IL1 Ra in experimental arthritis required continuous infusion. Randomised clinical trials in RA have also demonstrated modest anti-inflammatory effects and significant slowing in the rate of joint damage. The reduced joint damage manifested initially as slowing in the rate of cartilage surface loss and, subsequently, as inhibition of cartilage and bone invasion by proliferating synovial pannus. These effects may be in part attributable to reduced IL1 mediated mononuclear cell infiltration of synovial tissue and protease release by intimal layer synoviocytes and macrophages.

1 Harris ED. Rheumatoid arthritis: pathophysiology and mplications for therapy. N Engl J Med 1990;322:1277-89.

2 Arend WP, Dayer J-M. Inhibition of the production and effects of interleukin-1 and tumor necrosis factor $\alpha$ in rheumatoid arthritis. Arthritis Rheum 1995;38:151-60.

3 Dinarello CA. Biologic basis for interleukin-1 in disease. Blood 1996; 87:2095-147.

4 van den Berg WB. Lessons for joint destruction from animal models. Curr Opin Rheumatol 1997; 7:221-8.

5 Joosten LAB, Helsen MMA, van de Loo FAJ, van den Berg WB. Anticytokine treatment of established type II collageninduced arthritis in DBA $/ 1$ mice. A comparative study using anti-TNF $\alpha$, anti-IL- $1 \alpha / \beta$ and IL-1Ra. Arthritis Rheum 1996;39:797-809.

6 Bakker AC, Josten LAB, Arntz OJ, et al.Gene therapy of murine collagen-induced arthritis : Local expression of the human IL-1ra protein prevents onset. Arthritis Rheum 1997;40:893-900.

7 Drevlow BE, Lovis R, Haag MA, et al. Recombinant human interleukin-1 receptor type I in the treatment of patients with active rheumatoid arthritis. Arthritis Rheum 1996;39: with active

8 Campion GV, Lesback ME, Lookabaugh J, Gordon G, Catalano $M$ and the IL-1ra Arthritis Study Group. Dose -range and dose-frequency study of recombinant human interleukin-1 receptor antagonist in patients with rheumainterleukin-1 receptor antagonist in patients with $\mathrm{r}$
toid arthritis. Arthritis Rheum 1996;39:1092-101.

9 Bresnihan B, Alvaro-Gracia JM, Cobby M, et al. Treatment of rheumatoid arthritis with recombinant human interleukin-1 receptor antagonist. Arthritis Rheum 1999; 41:2196-204.

10 Felson DT, Anderson JJ, Boers M, et al. The American College of Rheumatology preliminary core set of disease activity measures for rheumatoid arthritis clinical trials. Arthritis Rheum 1993;36:729-40.

11 Paulus HE, Egger MJ, Ward JR, Williams HJ and the Cooperative Systematic Studies of Rheumatic Diseases Group. Analysis of improvement in individual rheumatoid arthritis patients treated with disease-modifying antirheumatic drugs, based on findings in patients treated with placebo. drugs, based on findings in patients

12 Nuki G, Rozman B, Pavelka K, Emery P, Lookabaugh J, Musikic P. Interleukin-1 receptor antagonist continues to demonstrate clinical improvement in rheumatoid arthritis. Arthritis Rheum 1997;40:S224

13 Larsen A, Dale K, Eek M. Radiographic evaluation of rheumatoid arthritis and related conditions by standard reference films. Acta Radiol Diagn 1977;18:481-91.

14 Jiang Y, Genant HK, Watt I, Cobby M, Bresnihan B, Aitchison $\mathrm{R}$, et al. A multicentre, double-blind, dose ranging, randomized and placebo controlled study of recombinant human interleukin-1 receptor antagonist in patients with rheumatoid arthritis: radiological progression and correlation of Genant and Larsen scoring methods. Arthritis Rheum (in press).

15 Bendele A, McAbee T, Sennello G, Fraizier J, Chlipala E, McCabe D Efficacy of sustained blood levels of interleukin-1 receptor antagonist in animal models of arthritis. Arthritis Rheum 1999; 42:498-506.

16 Cunnane G, Cassidy N, Madigan A, Murphy E, FitzGerald $\mathrm{O}$, Bresnihan $\mathrm{B}$. The effects of treatment with interleukin-1 receptor antagonist on the synovial membrane and joints of patients with rheumatoid arthritis. Arthritis Rheum 1998; 41:S138. 